\title{
Left ventricular restoration surgery for isolated left ventricular noncompaction: Report of the first successful case
}

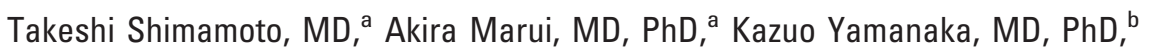
Norihiro Shikata, MD, ${ }^{\mathrm{c}}$ Keiichi Tambara, MD, PhD, ${ }^{a}$ Tadashi Ikeda, MD, PhD, ${ }^{a}$ and Masashi Komeda, MD, PhD, ${ }^{a}$ Kyoto, Japan

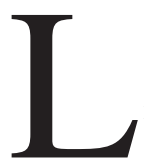
eft ventricular (LV) noncompaction (LVNC) is a rare congenital dysfunction of ventricular morphogenesis resulting from arrested muscle fiber compaction in early embryogenesis. We report a first successful case of left ventricular restoration (LVR) surgery in a patient with LVNC with severe LV dysfunction. LVR of septal anterior ventricular exclusion and concomitant mitral and tricuspid annuloplasty were successfully performed.

\section{Clinical Summary}

A 57-year-old woman had been followed up by a cardiology clinic for chronic heart failure with $40 \mathrm{mg}$ of oral furosemide. She was referred to our hospital because of worsening dyspnea and deteriorating hemodynamics in New York Heart Association class IV. Her symptoms were refractory to diuretic therapy. She had had permanent pacemaker implantation because of sick sinus syndrome and atrioventricular block 10 years earlier. A chest x-ray film showed pulmonary congestion with cardiomegaly. Findings on a coronary angiogram were not remarkable. An echocardiogram revealed diffuse LV hypokinesis and apicoseptal akinesis, 2 mobile apical thrombi $(36 \times 46 \mathrm{~mm}$ and $10 \times 7 \mathrm{~mm})$, and moderate mitral and tricuspid regurgitation. LV end-diastolic diameter was $58 \mathrm{~mm}$ and LV ejection fraction (LVEF) $22 \%$. There was no other associated congenital anomaly. Characteristically, LV trabeculations and intertrabecular recesses were prominent (Figure 1). There was thin, compacted myocardium on the epicardial side of the LV wall and thicker, noncompacted myocardium on the endocardial side. The ratio of noncompacted to compacted thickness was approximately 2.0 to 3.0 , which met the diagnostic criteria of isolated LVNC. ${ }^{1}$

Surgery was performed through a median sternotomy and the patient was placed on cardiopulmonary bypass. Removal of

From the Department of Cardiovascular Surgery, Kyoto University Graduate School of Medicine, ${ }^{\mathrm{a}}$ the Department of Cardiovascular Surgery, Koseikai Takeda Hospital, ${ }^{\mathrm{b}}$ and the Department of Cardiology, Second Min-iren Central Hospital, ${ }^{\mathrm{c}}$ Kyoto, Japan.

Received for publication Feb 2, 2007; revisions received March 5, 2007; accepted for publication March 15, 2007.

Address for reprints: Masashi Komeda, MD, PhD, Professor and Chairman, Department of Cardiovascular Surgery, Kyoto University Graduate School of Medicine, 54 Shogoin, Kawahara, Sakyo, Kyoto, Japan; 606-8507 (E-mail: komelab@kuhp.kyoto-u.ac.jp).

J Thorac Cardiovasc Surg 2007;134:246-7

$0022-5223 / \$ 32.00$

Copyright @ 2007 by The American Association for Thoracic Surgery doi:10.1016/j.jtcvs.2007.03.021 thrombi (Figure 2) and mitral and tricuspid annuloplasty with flexible rings were completed. LVR with the Dor endoventricular patch plasty was initially tried. When the LV was filled with blood, however, blood leakage was observed between the patch and deeply trabeculated myocardium. The Dor method was switched to septal anterior ventricular exclusion, in which an oval Dacron patch was sewn to the $\mathrm{LV}$ wall by using multiple transmural stitches to prevent blood leakage around the patch. Aortic crossclamp time was 64 minutes and cardiopulmonary bypass time was 92 minutes. Postoperative echocardiogram showed improved LV dimension and function (LV enddiastolic diameter $=43 \mathrm{~mm}, \mathrm{LVEF}=32 \%$ ) with no mitral and tricuspid regurgitation. There was no leakage around the Dacron patch to the excluded cavity. She was discharged in very good condition on postoperative day 43. She is doing well in New York Heart Association class I with $20 \mathrm{mg}$ of oral furosemide 6 months after the operation with an LV end-diastolic diameter of $45 \mathrm{~mm}$ and an LVEF of $33 \%$.

\section{Discussion}

To our knowledge, this is the first report of successful LVR surgery in a patient with LVNC. LVNC is a rare congenital cardiomyopathy resulting from intrauterine arrest of compaction of the loose interwoven meshwork. ${ }^{1}$ There is a high rate of other coexisting congenital cardiac anomalies such as ventricular septal defect and LVNC, with no other congenital anomaly being defined as isolated LVNC. ${ }^{2}$ Because of the poorly developed myocardium, patients with a severe form of the disease often die in the neonatal period. The majority of adults with LVNC show enlarged LV end-diastolic diameter and reduced LVEF at initial presentation. ${ }^{1,3,4}$ Diagnosis is made by echocardiogram. Multiple trabeculations and deep recesses in communication with the ventricular cavity are present and the ratio of noncompacted to compacted myocardial thickness is more than 2. ${ }^{1,3}$ Apical and midventricular 


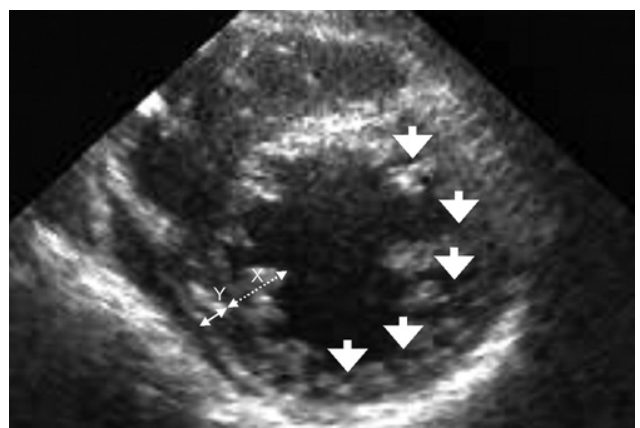

Figure 1. Short-axis view of the echocardiogram. Note that LV trabeculation and intertrabecular recesses were prominent (white arrows). The ratio of noncompacted $(X)$ to compacted $(Y)$ thickness of myocardium was 3 in the inferior wall. Ratios ranged between 2 and 3 according to each LV segment.

segments of both inferior and lateral walls are commonly affected in more than $80 \%$ of patients with $\mathrm{LVNC},{ }^{1}$ and all noncompacted segments are hypokinetic. The prevalence ranges from $0.05 \%$ to $0.24 \%$ per year among echocardiographic databases. ${ }^{3}$ Valvular structure is not usually affected, although malformation of papillary muscles and abnormal chordal attachment to valve leaflet are occasionally observed in severe forms of LVNC. ${ }^{2,4}$ Functional mitral regurgitation owing to annular dilatation is commonly associated with LVNC. ${ }^{4}$ Patients with LVNC have a poor prognosis because LV systolic function is gradually depressed with lethal complications such as ventricular arrhythmias and thromboembolism. ${ }^{1,3}$ There is no effective therapy for this disease; thus, treatment is restricted to alleviation of the symptoms and complications such as an implantable cardioverter-defibrillator. ${ }^{1}$ The only surgical treatment so far reported is heart transplantation. ${ }^{1}$

There were various new findings in this case. First, LVR surgery was effective in treating LVNC associated with large LV dimension and depressed LVEF by eliminating the embolic source, reducing $\mathrm{LV}$ diameter and improving LVEF by excluding the diseased LV area. LVR maintained its effectiveness with improved LVEF, reduced LV end-diastolic diameter, and reduced requirement of oral furosemide at 6 months' follow-up. This operation may be particularly effective in cases in which demarcation be-

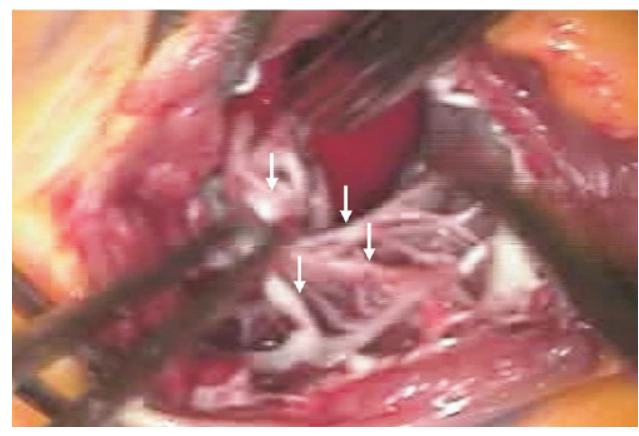

Figure 2. Intraoperative view of prominent trabeculations through the left ventriculotomy. A Dacron patch were sewn to exclude apicoseptal walls with deep trabeculations (white arrows) through this surgical view.

tween the compacted and noncompacted segment is evident. The fitting between the myocardium and Dacron patch can easily be loose because noncompacted myocardium is trabeculated and fragile. We believe the method of using transmural LV stitches is useful. Second, mitral and tricuspid annuli of this case were dilated and their papillary muscles had no abnormalities, with normal choral attachments to the leaflets. Therefore, we selected annuloplasty rings to treat those regions. Mitral and tricuspid regurgitation associated with LVNC can be treated with annuloplasty rings effectively unless they have abnormal subvalvular apparatus and organic leaflet change.

\section{References}

1. Oechslin EN, Attenhofer Jost CH, Rojas JR, Kaufmann PA, Jenni R. Long-term follow-up of 34 adults with isolated left ventricular noncompaction: a distinct cardiomyopathy with poor prognosis. J Am Coll Cardiol. 2000;36:493-500.

2. Burke A, Mont E, Kutys R, Virmani R. Left ventricular noncompaction: a pathological study of 14 cases. Hum Pathol. 2005;36:403-11.

3. Stollberger C, Finsterer J. Left ventricular hypertrabeculation/noncompaction. J Am Soc Echocardiogr. 2004;17:91-100.

4. Sengupta PP, Mohan JC, Mehta V, Jain V, Arora R, Pandian NG, et al. Comparison of echocardiographic features of noncompaction of the left ventricle in adults versus idiopathic dilated cardiomyopathy in adults. Am J Cardiol. 2004;94:389-91. 